\title{
Self-Care Agency Power Components Among Patients with Heart Failure: A Qualitative Directed Content Analysis Based on the Orem Self-Care Theory
}

\author{
Ghasem Abotalebidariasari, ${ }^{1}$ Robabe Memarian,, ${ }^{2,}$ Zohreh Vanaki, ${ }^{3}$ Anoshirvan Kazemnejad, ${ }^{4}$ and \\ Nasim Naderi ${ }^{5}$ \\ ${ }^{1}$ PhD Student of Nursing, Department of Nursing, Faculty of Medical Sciences, Tarbiat Modares University, Tehran, Iran \\ ${ }^{2}$ Assistant Professor, Department of Nursing, Faculty of Medical Sciences, Tarbiat Modares University, Tehran, Iran \\ ${ }^{3}$ Associate Professor, Department of Nursing, Faculty of Medical Sciences, Tarbiat Modares University, Tehran, Iran \\ ${ }^{4}$ Professor, Department of Biostatistics, Faculty of Medical Sciences, Tarbiat Modares University, Tehran, Iran \\ ${ }^{5}$ Associate Professor Of Cardiology , Fellowship of Heart Failure and Transplantation, Rajaie Cardiovascular Medical and Research Center, Iran University of Medical Sciences, \\ Tehran, Iran \\ "Corresponding author: Robabe Memarian, Assistant Professor, Department of Nursing, Faculty of Medical Sciences, Tarbiat Modares University, Tehran, Iran. Tel: \\ +98-2182883554, E-mail: memari_r@modares.ac.ir
}

Received 2016 September 01; Revised 2016 September 15; Accepted 2016 October 29.

\begin{abstract}
Background: Self-care is a self-regulatory action, which necessitates self-care power.

Objectives: The current study aims at exploring self-care agency power components among patients with heart failure by the Orem self-care theory. Methods: The current qualitative study utilized content analysis approach based on the Orem self-care theory. Twenty-seven patients with heart failure and their family members were purposefully recruited from Shahid Rajaie cardiovascular center, Tehran, Iran. The study data were collected from December 2014 to May 2015 through in-depth semi-structured interviews. Data collection was continued until data saturation which was attained after the 24th interviews and hence, the last 3 interviews produced no new data. Data analysis was carried out by the directed content analysis approach proposed in 2005 by Hsieh and Shannon.

Results: Data analysis yielded 79 primary codes, which represented the instances of self-care agency power components among patients with heart failure. The main components of self-care agency power were ability to acquire self-care knowledge, ability to identify factors aggravating heart failure, motivation for self-care, physical ability to engage in self-care activities, ability to adjust physical activities in order to save energy, ability to monitor bodily functions, reasoning about the causes of related symptoms, ability to decide on appropriate self-care measures, ability to understand the nature of the disease, ability to communicate with others to use their capabilities for self-care, ability to organize self-care measures, and ability to adhere to self-care activities. The most basic abilities are self-care knowledge, physical ability, and self-care motivation.

Conclusions: The findings of the study indicated that effective self-care among patients with heart failure requires a wide range of self-care abilities. Nurses can use the findings to develop self-care plans for patients with heart failure.
\end{abstract}

Keywords: Self-Care Agency Power Components, Heart Failure Orem Self-Care Theory

\section{Background}

Heart failure (HF) is among the most prevalent chronic diseases worldwide (1) and a major healthcare challenge (2). About $1 \%-2 \%$ of adults in the developed countries have HF (3). HF is also among the most common causes of hospital bed occupancy (4). The manifestations of HF, such as dyspnea, fatigue, edema, and activity intolerance impair the activities of daily life (5) and result in frequent hospitalization (2). Frequent hospitalization, in turn, imposes heavy costs on healthcare systems (6), which is equal to about $1 \%-2 \%$ of total health budget (1).

Self-care is the core component of HF management (7). The guideline of the European society of cardiology suggests both pharmacological and non-pharmacological therapies for HF (8). Despite great advances in recent years, sole pharmacological therapies do not completely manage HF (9). Therefore, non-pharmacological therapies are needed for effective HF management. Nonpharmacological therapies are mainly based on self-care (8), which has a significant role in decreasing hospitalization and promoting health (6). Self-care activities for patients with HF include secondary prevention measures (such as limited salt and fluid intake and daily weight assessment) and deciding upon the need for medical care (for instance asking for medical help once experiencing symptoms) (10). However, despite the significant role of self-care in effective management of HF, most previous studies reported that patients' self-care was poor $(7,11-13)$. According to Riegel et al., one of the reasons behind poor self-care ability of patients with HF is their poor power to do highly-complex HF-related self-care activities (14).

In her self-care deficit theory, Orem defined self-care as a regulatory activity whose prerequisite is to have the

Copyright (c) 2016, Baqiyatallah University of Medical Sciences. This is an open-access article distributed under the terms of the Creative Commons 
power to do self-care (15). She also defined self-care agency power as the ability to identify factors affecting health and function, the ability to decide upon self-care activities to regulate the function, and the ability to perform selfcare activities to fulfill self-care requisites. According to Orem, self-care agency power components (SCAPCs) is an absolute requirement to show such behaviors (16). SCAPCs range from knowing about oneself as a self-care agent to the abilities to maintain energy, motivation, and body position as well as the abilities to establish communication and integrate self-care abilities into the changing personal, familial, and social life (17).

Many studies are conducted so far on patients with HF to identify the barriers and the facilitators to self-care. Siabani et al. performed a meta-synthesis and found selfcare knowledge, long history of HF, a supportive environment, and spirituality as the main facilitators of self-care. They also reported that the barriers to self-care are functional limitations, complexity and severity of the underlying problem, depression, cultural beliefs, cognitive decline, inappropriate educational programs, poor communication skills, and social isolation (18). Holden et al. also found that numerous factors can limit self-care activities which include, but not limited to, simultaneous affliction with another chronic condition, complexity of self-care activities, shortage of resources, insurance-related problems, poor weather conditions, and limited self-care knowledge (19).

Most previous studies dealt mainly with the effects of demographic and environmental factors on patients' selfcare. Consequently, despite Orem's great emphasis on the role of SCAPCs on engagement in self-care activities, only few studies are conducted to address it. Understanding SCAPCs among patients with HF can help nurses identify patients' weaknesses and strengths and develop strategies to improve their self-care power.

\section{Objectives}

The current study aims at exploring SCAPCs among patients with HF by the Orem self-care theory.

\section{Methods}

It was a descriptive study. A purposive sample of 24 patients with HF and 3 family members were recruited from Shahid Rajaie cardiovascular, medical, and research center, Tehran, Iran.

The study data were collected from December 2014 to May 2015 through in-depth semi-structured interviews. The interviews were held face to face and personally by asking predetermined questions developed based on the SCAPCs construct. Two sample questions were 'How did you learn about caring for yourself?' and 'What factors enhance your motivation for self-care?' In addition, probing questions were asked for thorough exploration of participants' experiences. The length of the interviews was 45 minutes, on average. Data collection was continued until reaching data saturation. Data achieved after conducting 24 interviews and the last 3 interviews yielded no new data. All interviews were recorded and then typed verbatim. The transcript of each interview was read several times to understand its content and then analysis was started.

The data were analyzed by the directed content analysis approach introduced in 2005 by Hsieh and Shannon (20). This approach is used in cases where the researcher wishes to retest existing theory in a new context (21). Initially the concept of SCAPCs was extracted from the Orem theory and used as the basic concept for data analysis. Then, a working definition of the concept was provided based on the Orem theory and its 10 sub-concepts were identified as follows:

1. Ability to acquire technical knowledge about selfcare from authoritative sources, to retain it, and to operationalize it

2. Ability to maintain attention and exercise requisite vigilance with respect to self as self-care agent and internal and external conditions and factors significant for self-care

3. Ability to control the position of the body and its parts in the execution of the movements required for the initiation and completion of self-care operations

4. Controlled use of available physical energy sufficient to initiate and continue self-care operations

5. Ability to reason within a self-care frame of references;

6. Ability to make decisions on of self-care-and operationalize the decisions

7. Motivation for self-care

8. Ability to order discrete self-care actions or action systems into relationships with prior and subsequent actions toward the final achievement of regulatory goals of self-care

9. Ability to consistently perform self-care operations and integrate them with relevant aspects of personal, familial, and social life

10. A repertoire of cognitive, perceptual, manipulative, communicative, and interpersonal skills adapted to the performance of self-care operations (16).

These sub-concepts were considered as categorization matrix for data analysis. After developing a categorization matrix, all the data (interviews) were reviewed for content and coded for correspondence with or exemplification of the identified categories. Accordingly, excerpts from in- 
terview transcripts, which fitted the categories, were identified and coded. The codes were placed into the predetermined categories, i.e. the abovementioned 10 subconcepts. Data analysis yielded 79 primary codes that represented the instances of self-care agency power components among patients with HF. The 79 primary codes were categorized into 10 subcategories.

The criteria proposed by Lincoln and Guba (22) were adopted to ensure the reliability of the data. To establish the credibility of the data, several techniques were used including maximum variation sampling (in terms of participants' gender, age, residence, HF class, employment, and past medical history), prolonged engagement with the participants (more than 6 months), and member checking. During the process of member checking, the codes of each interview were provided to the corresponding interviewee and he/she was asked to determine if the codes fitted his/her experiences. The validity of the data was also maintained by the peer checking technique. Accordingly, the accuracy of coding and code allocation to the categories were assessed. Table 1 shows examples of coding and categorizing.

The current study was approved by the ethics committee and the institutional review board of Tarbiat Modares university, Tehran, Iran (approval code number: D7035/52; February 26, 2014). The study goal was explained to the participants at the beginning of each interview. Then, they were ensured that participation in the study was voluntary and that their data would remain confidential. Finally, informed consent was obtained from the participants.

\section{Results}

The mean age of the participants was $50.22 \pm 15$ years, on average. Most of the participants were male (63.3\%), with class IV HF (37.5\%), and held high school diploma (25\%). In total, 27 interviews were conducted out of which 79 primary codes were extracted and categorized into the 10 SCAPCs.

\subsection{Ability to Acquire Self-Care Knowledge from Authoritative Sources}

The participants attempted to acquire self-care knowledge to understand their underlying disease and its contributing factors and make decisions and judgments about self-care. Greater knowledge about their underlying disease helped them identify their own self-care requisites and take measures to fulfill them. They acquired knowledge both empirically and non-empirically. Most of the participants acquired self-care knowledge from authoritative sources such as physicians, nurses, pamphlets, medical textbooks, the internet, and television.
Once there is something unknown to me, I refer to hospital and ask my doctor about it (a 63-year-old male). The hospital provided us with a series of brochures which I studied and understood what I needed to do (a 35-year-old male).

On the other hand, patients with a long history of HF noted that they empirically learned over time about how to care for themselves.

I have hadthis disease throughout my life, my [selfcare] knowledge is based on my own personal experiences. For instance, if I did intense physical activity, I experienced problems and thereby, I would understand that intense activity could be harmful to me (a 26-year-old male).

On the other hand, the participants with limited selfcare knowledge experienced difficulties in doing self-care activities. For instance, a 52-year-old male said,

I don't know how much water I can drink. My doctor did not say anything to me about it.

4.2. Ability to Maintain Attention and Exercise Requisite Vigilance with Respect to Self as Self-Care Agent, Internal and External Conditions, and Factors Significant for Self-Care

After acquiring self-care knowledge, the participants identified factors affecting their health and attempted to avoid them through following proper precautions. For instance, they noted that common cold could aggravate their conditions and hence, they prevented themselves from developing common cold through adopting appropriate prevention strategies.

I avoid going to very crowded places to prevent common cold. Moreover, I receive flu vaccine each year (a 23year-old female).

They also noted that they avoided risk factors such as weight gain, intense physical activity, high-salt and highfat foods, poor weather conditions, stressors, and strong odors.

I eat salt-free and low-fat foods (a 50-year-old male). As obesity is a risk factor for the disease, I reduced my weight by 15 kilograms (a 62-year-old female).

\subsection{Ability to Control Physical Activities to Initiate and Com- plete Self-Care Activities}

Another basic skill for self-care was physical ability to engage in self-care activities. In the initial phases of HF (i.e. class I and II HF), the participants did not experience significant problems to do self-care activities due to having mild symptoms, if any. Patients with class I and II HF mentioned that they had no limitation to do physical activity and were physically able to perform self-care activities.

I have no limitation to do activities and hence, I care for myself on my own. I never feel ill at all. I have no shortness 
Table 1. Examples of Coding and Categorizing the Data

\begin{tabular}{lll}
\hline Participants' Quotations & Primary Codes & Categories of SCAPCs \\
\hline $\begin{array}{l}\text { I've read most of the books which are about my } \\
\text { disease such as this 'cardiovascular diseases' } \\
\text { book. }\end{array}$ & Acquiring self-care knowledge from books & Acquiring knowledge from authoritative sources \\
\hline $\begin{array}{l}\text { I try to climb the stairs very slowly. Once } \\
\text { climbing stairs, I take a rest after climbing some } \\
\text { stairs and then climb some more stairs. }\end{array}$ & Taking a rest between activities & $\begin{array}{l}\text { Controlled use of available physical energy to } \\
\text { initiate and continue self-care activities }\end{array}$ \\
\hline $\begin{array}{l}\text { I like to get better and do not suffer agony. I also } \\
\text { like my mother and family members to be free } \\
\text { of difficulty. }\end{array}$ & $\begin{array}{l}\text { Doing self-care in order to prevent family members } \\
\text { from experiencing difficulty }\end{array}$ & Motivation for self-care \\
\hline
\end{tabular}

Abbreviation: SCAPCs, self-care agency power components.

of breath and can climb stairs easily. I even go mountain climbing (a 52-year-old male).

However, disease progression and symptom aggravation, particularly the development of fatigue and dyspnea, severely restricted the patients' ability to do physical and self-care activities. Patients with advanced phases of HF (i.e. class III and VI HF) were even unable to move and do selfcare activities and were dependent on others for self-care.

Physical disability prevents one from doing physical activities. I would care for myself if I had the power; however, I don't. Sometimes, I'm so ill that I can't even cover myself with a blanket (a 60-year-old male).

\subsection{Controlled use of Available Physical Energy Sufficient to Ini- tiate and Continue Self-Care Activities}

Limited ability and power for physical activity due to disease aggravation required the patients to adjust their activities to prevent fatigue and save energy. Subsequently, they preferred to stay at home more and take a break.

I don't do intense activities to prevent fatigue (a 26year-old male). Most of the times, I rest at home (a 62-yearold male).

Fatigue and dyspnea also prevented the patients from doing sport. Therefore, they gave up sport. The patients also noted that they attempted to do their activities intermittently and to take a rest in the midst of their activities.

I experienced shortness of breath once doing sport; therefore, I avoided going to the gym (a 29-year-old male). Once climbing stairs, I take a break after climbing some stairs and then climb some more stairs (a 65-year-olf male).

Some of the patients, particularly blue-collar workers, were even compelled to stop working or adjust their occupational activities due to experiencing fatigue and dyspnea at work.

I was a worker at a papermaking factory. However, it is for 4 years that I do not go to work (a 50-year-old male).

\subsection{Ability to Reason Within a Self-Care Frame of References}

Through a knowledge-based reasoning ability, patients with HF can monitor their bodily functions and judge id they are normal. Participants of the current study also mentioned that they diagnosed abnormalities in their body through monitoring their body weight, urine output, and vital signs regularly. Reasoning ability also helps patients with cardiovascular diseases identify causes of abnormalities and make sound decisions to remove them. A 26-year-old female said, Each day, I measure the volume of my urine and watch whether it has increased or decreased. If it is less than the normal volume, I ask myself about the reason and compare the output with my fluid input. Moreover, when I experience weight gain, I ask myself, 'Why has my weight increased?'

However, some patients did not have the reasoning ability and hence, they could not achieve an accurate understanding about the reasons behind developing new symptoms. Therefore, they adopted strategies that did not alleviate their symptoms, but aggravated them.

When I develop coughing at nights once I'm asleep, I immediately understand that it is due to the dryness of my throat. Therefore, I get up and drink a glass of water (a 52year-old male).

\subsection{Ability to Make Decisions on Self-Care and Operationalize the Decisions}

Decision making ability allowed the participants to make the best possible decisions on self-care based on their own physical conditions and accessible resources. Moreover, such an ability helped them make sound decisions to avoid risks once they encountered them.

Whenever the air is polluted, I avoid going out or I use facemask if I have to (a 26-year-old male).

Moreover, decision making ability helped the participants adopt the most effective strategies to alleviate their symptoms based on the situation. 
I use oxygen once I develop shortness of breath at home. In outdoor places, I manage shortness of breath using spray. If these strategies do not help, I immediately go to hospital (a 62-year-old male).

On the other hand, participants who lacked decision making ability were unable to manage their problems and symptoms.

I do nothing once I experience shortness of breath. Nothing can help in such situations. It should be relieved spontaneously because we can do nothing (a 75-year-old male).

\subsection{Motivation for Self-Care}

Patients motivated for self-care, consciously and deliberately engage in doing self-care activities. Most of the participants' motives for self-care were personal. In other words, they engaged in self-care activities to gain personal benefits such as saving their own lives, alleviating their own pain, achieving recovery, and regaining health.

My hope is stem cells. I hope science advances to such an extent that I will be able to return to life by stem cells because I like my life (a 62-year-old male).

Some participants had spiritual motivation for selfcare. Relying on God and believing that none of God's actions is fruitless helped this group of patients gain mental calmness and improved their morale for self-care.

Praying and relying on God have boosted my morale. I'm extremely calm. Such calmness helps me care for myself with a higher morale (a 23-year-old female).

Some other participants' motives for self-care were familial. In other words, they engaged in self-care activities to boost their family members' morale and also avoid inflicting pain and agony on them.

I care for myself mostly for the sake of my parents. I see that they are exhausted and experience difficulties because of me and hence, I try hard to get recovery and prevent them from enduring agony (a 23-year-old female).

4.8. Ability to Consistently Perform Self-Care Activities and Integrate Them with Relevant Aspects of Personal, Familial, and Social Life

To improve their physical condition and prevent the progression of their disease, the study participants had made some lifestyle modifications and attempted to adhere to the modifications. For instance, they had restricted the intake of salt, fat, and fluids, reduced their body weight, increased their physical activity, and managed their stress.

It is now 3 years that I have never used salt, oil, fried foods, and colas and have strictly followed my treatment and dietary regimens (a 56-year-old male).
However, the progressive nature of HF, the complexity of treatment and dietary regimens, and the little likelihood of complete recovery had caused the participants severe fatigue and discouraged them from doing self-care activities and also following their regimens.

I'm desperately tired. Primarily, I was very eager and thought that I would continue my normal life through following treatment and dietary regimens. But, once the condition got worse, I became desperate and gave up everything (a 35-year-old male).

\subsection{Ability to Order Discrete Self-Care Actions or Action Systems to Relate Prior Actions to Subsequent Actions}

The participants tried to enhance the effectiveness of self-care measures through ordering and sequencing them. Accordingly, they monitored their bodily functions, diagnosed deviant functions, decided on the necessary measures, implemented measures, and evaluated outcomes.

I measure my fluid intake and body weight. In case of any increased fluid intake, I use furosemide pills to increase my urinary output (a 26 -year-old male).

Because of senility and comorbidity, the study participants needed to use different medications. Consequently, they tried to prevent gastrointestinal side effects of their medications and drug interactions through developing a medication plan.

Medications had irritated my stomach and hence, I increased the number of meals and decreased the amount of them. This strategy improved my condition very much (a 26-year-old male).

However, patients' inability to organize self-care measures worsened their symptoms.

I take 14 pills a day. The pills irritate my stomach. To prevent stomach problems, I drink great amount of water, which is harmful to my heart (a 52-year-old male).

4.10. A Repertoire of Cognitive, Perceptual, Manipulative, Communicative, and Interpersonal Skills to Perform Self-Care Activities

At the beginning of their disease, the participants did not care for themselves effectively due to having limited knowledge about the chronic nature of their disease. Initially, they did not know that their disease was a chronic condition. However, they gradually acquired knowledge and understood that they were afflicted by a chronic condition. Consequently, they engaged in self-care activities more actively.

I was shocked once they told me that I have heart failure. I had no information about the disease and thought that it was just a fluctuation in my heart rate which would 
be corrected by some pills. But, I have understood my condition by now. Currently, I know what my problem is and what I should do (a 23-year-old female).

The study participants also attempted to communicate with others and ask for others' help once their problems got worse and they were unable to care for themselves. Besides, alleviating disease-related stress, communication with others (such as family members) also improved their morale.

When I feel seriously ill, I call my daughter. She knows what to do for me (a 75-year-old male). When I'm under stress, I talk with my family members. They help and console me very much (a 23-year-old female).

\section{Discussion}

The main SCAPCs among participants with HF were ability to acquire self-care knowledge both empirically and non-empirically, ability to identify factors aggravating $\mathrm{HF}$, personal and familial motivation for self-care, physical ability to engage in self-care activities, ability to adjust physical activities to save energy, ability to monitor bodily functions and also adopt reasoning about the causes of symptoms, ability to decide on and choose the most appropriate self-care measures based on the available resources and the immediate conditions once encountering symptoms and HF risk factors, ability to understand the nature of the disease, ability to communicate with others to use their capabilities for self-care, ability to organize self-care measures, and ability to adhere to self-care activities.

The study findings revealed that one of the key SCAPCs was the ability to acquire self-care knowledge. Siabani et al reported that the lack of knowledge in patients with congestive heart failure (CHF), especially regarding diet and salt restriction, and misconceptions about CHF and its symptoms lead to failure of understanding the relationship between disease and symptoms (18). Orem noted that once experiencing changes in therapeutic self-care demand, an individual will show new self-care requisites. Accordingly, he/she will need greater self-care knowledge to make judgments and decide on self-care measures (16). It was also found that self-care knowledge helped the participants make judgments about the credibility of the measures they adopted to satisfy their self-care demands.

The findings of the present study also indicated that the participants paid attention to personal and individual factors affecting self-care. According to Orem, personal and environmental events and conditions affect self-care requisites and self-care agency power and hence, people can effectively perform self-care measures only when they have adequate knowledge about themselves and their environmental conditions. She also noted that knowledge about internal and external events and conditions helps people observe, give meaning to their observations, and relate the meanings of events and conditions to potential measures (16).

The study also found that patients with HF needed motivation for self-care. Orem believes that besides knowledge, motivation is also an absolute prerequisite to do selfcare activities. In other words, people's lack of perseverance with doing self-care activities is due to their purposelessness and ignorance of the value of self-care. Personal and familial motivation of the study participants also enhanced their adherence to self-care activities. Orem also noted that decisions on self-care may be made based on the meaning of self-care activities to significant others. For instance, an individual may decide on doing self-care activities to make his/her family members glad (16). According to the results of the study by Mohammadpour et al., lack of sufficient motivation to take care of oneself was the main cause of poor self-care in $42 \%$ of patients with myocardial infarction (23).

The participants with severe HF experienced self-care deficit. Physical disability among patients with severe HF significantly reduced their cardiac output and tissue perfusion. Accordingly, they were compelled to restrict their physical mobility to save their energy. Holden et al. also reported that impaired physical mobility restricts self-care agency power (19). According to Orem, limited physical mobility prevents individuals from performing self-care activities and leaves them with self-care deficit (16).

The study findings also showed that patients with HF organized their self-care measures. It seems that they did so to handle the complexities of their cardiac problems and treatment regimens. Organization of care can prevent the complications of HF and the side effects of treatment regimens and enhance the effectiveness of self-care measures.

The study also found that the participants were aware of their physical conditions and had good communication skills for self-care. According to Orem, awareness of one's own condition helps individuals understand the meaning and the value of self-care. Such an understanding, in turn, facilitates rational and reasonable decision making on selfcare (16). Moreover, accurate understanding of one's own condition helps patients who are unable to care for themselves use family and community resources to perform self-care activities.

\subsection{Conclusion}

The findings of the current study indicated that patients with HF need different abilities to effectively perform self-care activities. The most basic abilities are selfcare knowledge, physical ability, and self-care motivation. 
Self-care knowledge helps patients decide upon the most appropriate self-care measures based on their own personal and environmental conditions. Moreover, it allows them to clearly understand their underlying disease and physical conditions and organize self-care activities. Selfcare motivation also helps patients adhere to self-care activities while physical ability enables them to engage in the activities. These findings represent the SCAPCs among patients with HF. Nurses can use these findings to develop self-care plans for patients with HF.

\section{Acknowledgments}

The current study was part of a PhD dissertation in nursing in the faculty of medical sciences, Tarbiat Modares university, Tehran, Iran. Authors hereby thank the administrators of Tarbiat Modares university who financially supported the study, and the participants, their family members, the administrators, and the staffs of Shahid Rajaie cardiovascular, medical, and research center for their support during the study.

\section{Footnote}

Authors' Contribution: Ghasem Abotalebidariasari: designing the study, conducting individual interviews, analyzing the data, interpreting the findings, drafting and submitting the manuscript; Robabe Memarian: designing the study, analyzing the data, interpreting the findings, critically reviewing of manuscript, and supervising the study process; Zohreh Vanaki: designing the study, analyzing the data, interpreting the findings, and supervising the study process; Anoshirvan Kazemnejad: analyzing the data, interpreting the findings, and supervising the study process; Nasim Naderi: analyzing the data, interpreting the findings, and supervising the study process; all authors read and approved the final manuscript.

\section{References}

1. Oertle M, Bal R. Understanding non-adherence in chronic heart failure: a mixed-method case study. Qual Saf Health Care. 2010;19(6):e37. doi: 10.1136/qshc.2009.033563. [PubMed: 21127097].

2. Saccomann ICRS, Cintra FA, Gallani MCBJ. Factors associated with beliefs about adherence to non-pharmacological treatment of patients with heart failure. Revista da Escola de Enfermagem da USP. 2014;48(1):18-24. doi: 10.1590/s0080-623420140000100002.

3. De Maeyer C, Beckers P, Vrints CJ, Conraads VM. Exercise training in chronic heart failure. Ther Adv Chronic Dis. 2013;4(3):105-17. doi: 10.1177/2040622313480382. [PubMed: 23634278].
4. Agren S. Supportive care for patients with heart failure and their partners: A descriptive and interventional study. Sweden: linkoping; 2010.

5. Sundin K, Bruce E, Barremo AS. Elderly women's experiences of support when living with congestive heart failure. Int J Qual Stud Health Well-being. 2010;5 doi: 10.3402/qhw.v5i2.5064. [PubMed: 20640021].

6. Manwere A, Saburi G, Charumbira A, Mukona D, Zvinavashe M. The relationship between self-care practices and readmissions among adults with chronic heart failure. Oriental J Sci Res. 2013;2(2):6-15.

7. Strachan PH, Currie K, Harkness K, Spaling M, Clark AM. Context matters in heart failure self-care: a qualitative systematic review. J Card Fail. 2014;20(6):448-55. doi: 10.1016/j.cardfail.2014.03.010. [PubMed: 24735549].

8. Linga P. Self care in patients with heart failure Stockholm. ; 2012.

9. Larsen P, Pedersen PU. Stimulation to self-care in patients with heart failure: A quasi-experimental study. Nurse Educ Pract. 2013;4(3) doi: 10.5430/jnep.v4n3p143.

10. Hajduk AM, Lemon SC, McManus DD, Lessard DM, Gurwitz JH, Spencer FA, et al. Cognitive impairment and self-care in heart failure. Clin Epidemiol. 2013;5:407-16. doi: 10.2147/CLEP.S44560. [PubMed: 24187511].

11. Oksel E, Akbiyik A, Kocak G. FP32 Self-care behaviour analysis of patients with chronic heart failure. Eur J Cardiovasc Nurs. 2009;8:22. doi: 10.1016/s1474-5151(09)60071-2.

12. Daryasari GA, Karkezloo NV, Mohammadnejad E, Vosooghi MN, Kagi MA. Study of the self-care agency in patients with heart failure. Iran J Critic Care Nurs. 2012;4(4):203-8.

13. Shojaei F, Asemi S, Najaf Y, Hosseini F. Self-care behaviors in patients with heart failure. Payesh. 2009;8(4):361-9.

14. Riegel B, Dickson VV, Hoke L, McMahon JP, Reis BF, Sayers S. A motivational counseling approach to improving heart failure self-care: mechanisms of effectiveness. J Cardiovasc Nurs. 2006;21(3):232-41. [PubMed: 16699364].

15. Khaledi GH, Mostafavi F, Eslami AA, Rooh Afza H, Mostafavi F, Akbar H. Evaluation of the Effect of Perceived Social Support on Promoting Self-Care Behaviors of Heart Failure Patients Referred to The Cardiovascular Research Center of Isfahan. Iran Red Crescent Med J. 2015;17(6):e22525. doi: 10.5812/ircmj.22525v2. [PubMed: 26328063].

16. Orem D. Nursing: Concepts and practice. 6 ed. Louis Mosby; 2001.

17. Meleis AI. Theoretical nursing: development and progress. American J Nurs. 1985;85(12):1350.

18. Siabani S, Leeder SR, Davidson PM. Barriers and facilitators to selfcare in chronic heart failure: a meta-synthesis of qualitative studies. Springerplus. 2013;2:320. doi: 10.1186/2193-1801-2-320. [PubMed: 23961394].

19. Holden RJ, Schubert CC, Eiland EC, Storrow AB, Miller KF, Collins SP. Self-care Barriers Reported by Emergency Department Patients With Acute Heart Failure: A Sociotechnical Systems-Based Approach. Ann Emerg Med. 2015;66(1):1-12. doi: 10.1016/j.annemergmed.2014.12.031. [PubMed: 25616317] 12e 1-2.

20. Hsieh HF, Shannon SE. Three approaches to qualitative content analysis. Qual Health Res. 2005;15(9):1277-88. doi:10.1177/1049732305276687. [PubMed: 16204405].

21. Elo S, Kyngas $H$. The qualitative content analysis process. J Adv Nurs. 2008;62(1):107-15. doi: 10.1111/j.1365-2648.2007.04569.x. [PubMed: 18352969].

22. Anney V. Ensuring the quality of the findings of qualitative research: looking at Trustworthiness Criteria... JEmerging Trends Educat Res Policy Studies. 2014;5(2):272-81.

23. Mohammadpor A, Rahmati SN, Khosravan S, Alami A. Investigating the level of self-care and supportiv eeducative needs of patients with myocardial infarction, based on orem's model. J Res Health. 2013:3(4):489-96. 